\title{
A role for redox regulation in PDAC
}

\section{Cff \\ ....NRF2 is critical for cell proliferation and redox homeostasis in PDAC...}

The transcription factor nuclear factor erythroid-derived 2-like 2 (NFE2L2, also known as NRF2) is a redox master regulator that maintains pancreatic cancer proliferation by stimulating mRNA translation and mitogenic signalling, according to a new study published in Cell. This finding reveals new strategies for treating the highly lethal pancreatic ductal adenocarcinoma (PDAC).

PDAC is one of the leading causes of cancer-related deaths with a poor prognosis related to late-stage diagnosis and resistance to current therapies. Oncogenic KRAS mutations are the principal driver of PDAC but no chemical inhibitors exist for the most common KRAS mutations in PDAC. "Previously, using cell biological and genetic approaches to identify pathways that promote pancreatic intraepithelial neoplasia, we found that oncogenic KRAS expression induced an important regulator of redox control," reports first author Iok In Christine Chio. This regulator, NRF2, strongly stimulated proliferation and suppressed

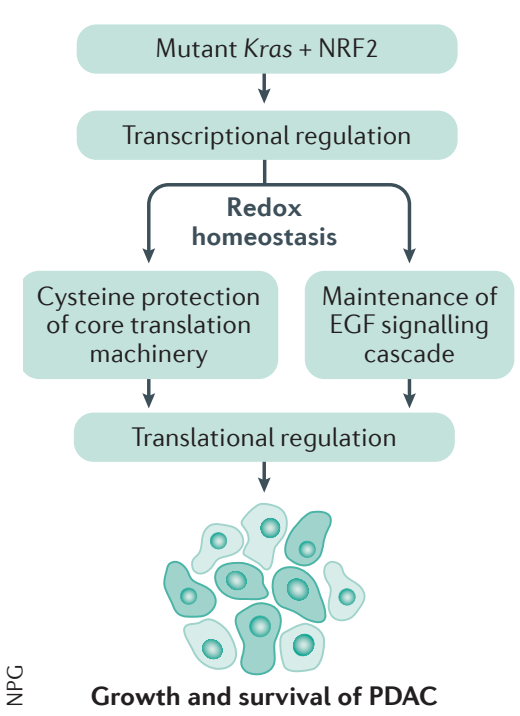

senescence of pancreatic cancer cells. "As transcription factors are notoriously difficult to target therapeutically, we sought to comprehensively analyse the mechanism through which NRF2 promotes pancreatic cancer," explains Chio.

The researchers first interrogated panels of normal and malignant human and mouse PDAC organoid models to investigate the role of NRF2 in both settings. NRF2 was found to be required for the survival of human tumour organoids, and knockdown of NRF2 impaired the proliferation of organoids in culture, as well as the orthotopic engraftment of malignant mouse organoids. Additionally, increased reactive oxygen species (ROS) levels were observed in tumour organoids following NRF2 ablation and antiproliferative defects were corrected by antioxidant administration. Thus, NRF2 is critical for cell proliferation and redox homeostasis in PDAC cells via the modulation of cytoplasmic ROS.

Oxidative stress can disrupt biological functions, but cellular redox reactions are also tightly regulated with essential roles as switches for signalling events. For example, cellular ROS can oxidize cysteine thiol groups, affecting the catalytic activity or conformation of the corresponding protein. Reactive cysteine-containing proteins were good candidates to carry out the redox-sensitive effector functions of NRF2, so the investigators examined changes in the cysteine proteome of mouse organoids depleted in NRF2 using a sensitive mass spectroscopy method. This analysis indicated the involvement of proteins participating in mRNA translation. Further functional analysis of NRF2-knockdown in a human pancreatic cancer cell line showed that this protein was required for cap-dependent and cap-independent mRNA translation. "This [effect] is through maintaining the reduced state of redox switches in the core translation machinery," explains Chio.

Furthermore, loss of NRF2 in Kras-mutant mouse organoids caused defects in autocrine mitogenic signalling pathways that regulate cap-dependent translation initiation, including the depleted shedding of epidermal growth factor (EGF). The metalloproteinase considered responsible for EGF shedding was also shown to be regulated in a redoxdependent manner, suggesting redox regulation of autocrine EGF receptor activation in Kras-mutant cells. Thus, the stimulatory effects of NRF 2 on EGF signalling and mRNA translation in PDAC both converge to promote global protein synthesis, ultimately supporting uncontrolled tumour growth and survival.

Combined inhibition of glutathione (an intracellular antioxidant) synthesis and an EGF receptor effector was shown to blunt pancreatic cancer growth in vitro and in vivo, indicating a potential novel therapeutic strategy. "Our work has provided new perspectives into the biochemical regulation of the translation apparatus and highlights the importance of protein synthesis in promoting pancreatic cancer," says Chio. As dysregulation of mRNA translation is a feature of all tumours, therapeutics that target protein synthesis could be promising anticancer drugs that overcome the genetic heterogeneity of tumours. The investigators are now planning to identify the most effective combinations of pro-oxidants and small molecule inhibitors to propose for clinical evaluation before further in vivo testing using genetic and orthotopic transplantation models of human organoids.

Iain Dickson

ORIGINAL ARTICLE Chio, I. I. et al. NRF2 promotes tumor maintenance by modulating mRNA translation in pancreatic cancer. Cell http://dx.doi.org/10.1016/j.cell.2016.06.056 (2016) 